\title{
Dimensiones de la territorialidad, ejes para un programa de educación ambiental en la ruralidad
}

\author{
Enriqueta del Carmen Caldera Reyes ${ }^{1}$ \\ Karla Patricia Dávila Castillo \\ Julio Cesar Laguna Gámez ${ }^{3}$
}

\section{RESUMEN}

La temática de este ensayo se centra en reafirmar la educación como una de las claves del desarrollo estratégico de todo territorio, de acuerdo a esto se presentan argumentos que con los cuales también se pueda aportar a la teoría del desarrollo rural, entre estos se asumen las experiencias en el territorio que aseguran validez de plantear y ejecutar propuestas educativas en la lógica de conservar el medio ambiente, en el caso de Matagalpa municipio tiene una cantidad de estudios productos de proyectos ejecutados en la ruralidad, especialmente en las unidades territoriales definidas como cuencas, particularmente se trabajará con dos de las cinco que se tienen en Matagalpa, para los cuales en la actualidad como parte de las etapas se organizará cada paso para demostrar la riqueza del trabajo con los comunitarios de Molino Norte y Jucuapa, según el desarrollo del territorio, para esto se determinan instrumentos como encuesta, entrevista y observación, que permitan obtener la información deseada. Importante es resaltar que toda la experiencia acumulada en estudios ya realizados en la temática de educación ambiental sirve de referencia para los nuevos planteamientos con los cuales se traten los desafíos de este siglo XXI.

Palabras clave: Territorio, cuenca, comunidad, educación y medio ambiente.

Recibido: 17 de noviembre de 2016

Aceptado: 16 de diciembre de 2016

1 Docente e investigadora FAREM-Matagalpa, UNAN-Managua. Correo electrónico: carcalre@yahoo.es

2 Docente e investigadora FAREM-Matagalpa, UNAN-Managua. Correo electrónico: kardycas@yahoo.es

3 Docente e investigador FAREM-Matagalpa, UNAN-Managua. Correo electrónico: jlagunagamez@yahoo.es 


\title{
Dimensions of territoriality, axes for an environmental education program in rurality
}

\begin{abstract}
The theme of this essay focuses on reaffirming education as one of the keys to the strategic development of all territories, according to which arguments are presented which can also be contributed to the theory of rural development. Experiences in the territory that ensure the validity of proposing and implement educational proposals in the logic of preserving the environment, in the case of Matagalpa municipality has a number of studies project products executed in rurality, especially in territorial units defined as basins, Particularly will work with two of the five that are in Matagalpa, for which currently as part of the stages will be organized every step to demonstrate the wealth of work with the community of Molino Norte and Jucuapa, depending on the development of the territory, To this end, instruments such as survey, interview and observation are determined so as to obtain the desired information. It is important to emphasize that all the experience accumulated in studies already carried out in the theme of environmental education serves as a reference for the new approaches that address the challenges of this 21 st century.
\end{abstract}

Keywords: Territory, watershed, community, education and environment. 


\section{INTRODUCCIÓN}

El desarrollo rural territorial con sus ejes y dimensiones es el corazón de este estudio, esta temática con todas las experiencias de intervención en los territorios da base para continuar con el ejercicio ya que como enfoque estratégico integral, presenta claramente las líneas dinamizadoras del territorio, según García (2000, p. 110-111), se evidencia la multiescalaridad, multisectorialidad, multitemporalidad, la ultiinstrumentalización todas parte de la multidimensionalidad territorial. En referencia a esto también Santos, (1996) retoma en su teoría que los elementos sociedad y naturaleza que al combinarse en el territorio generan sistemas de objetos y sistemas de acciones, que lo forman de modo inseparable, solidario y contradictorio, de acuerdo a esto retoma importancia de la temática a la luz de este siglo, siendo esencial la situación ambiental como un presente que lleva a entender la gestión de la cuenca como unidad territorial de desarrollo, específicamente las cuencas ubicadas en Matagalpa son las que se acompañarán, de ese proceso se tomará datos presentes y con la información, se harán valoraciones en la finalidad de aportar a la teoría rural del desarrollo un programa de educación ambiental que se formule a partir de las experiencias exitosas anteriores, y recoja temas reflejados en la teoría y práctica a manera de ejes válidos en formación y consolidación de los territorios.

\section{DESARROLLO}

Presentar la educación como un eje articulador de los diversos procesos del desarrollo humano sustentable, es lo que hacen Marúm-Espinoza y Reynoso-Cantú (2014), de hecho vinculan el término humano a la educación ya que como proceso genera el producto de un acto enseñar y aprender, dicho acto es muy propio de esta especie que ejercita de forma muy natural la informalidad y no formalidad de la educación, su énfasis es el aprendizaje como factor básico de cambio, ya que una vez que se adquiere la información y al aplicarla es producto de la construcción de significados según Ausubel, Novak, Hanesian (1983), en todo caso el cúmulo de símbolos al ejecutarse propicia cambios en la actividad social, esto indica que la educación es tan estratégica para entender que se dimensiona en el avance científico técnico de hoy, lo que sin lugar a dudas repercute en la situación ambiental.

Tomando lo expuesto anteriormente y asociado a la información proveniente de estudios realizados en la ruralidad, el Doctorado de Desarrollo Territorial Rural que sirve la UNAN FAREM Matagalpa, UNAN MANAGUA asume en su programa de formación aspectos educativos que propicien una reflexión genuina, comprometida de la situación en las que se manifiesta el desarrollo rural territorial, enfocado también a promover la responsabilidad social ante los recursos de la naturaleza.

En referencia a esta educación y reflexión de las condiciones en que está la naturaleza rural, Matagalpa se analiza desde la concepción de que el territorio es la materialización de la existencia humana en su espacio social Lefebvre, (1991, p 202), por ello es que en la búsqueda de materializar 
con elementos concretos reales se define el Plan de Ordenamiento Territorial AMUPNOR (2013), entre los tantos elementos naturales se clasifica los recursos hídricos presentándolos en sistema de cuencas, las que clasifica de la siguiente manera: 5 sub cuencas y 69 microcuencas ubicadas en una extensión de 64,305.15 ha, de las cuales las más importantes son el Rio Molino Norte y San Francisco, éstas cuentan con potencial hidrológico y de gestión de riesgo, lo que determina una cultura de vida que se ha venido construyendo pero que se trata de crear condiciones y acompañar desde el gobierno municipal, dichas potencialidades son manejable por la población Di Castri, F. (1995), las riquezas presentes están asociadas a paisaje, espacio, ambiente, escala, accesibilidad, sociedad, naturaleza y tiempo, todas interrelacionadas en el entramado social y natural de la cuenca, por su peso en contenido teórico práctico son calificadas en inter y transdisciplinarias.

A la luz de la teoría es válido entonces tomar las características rurales de las cuencas de Matagalpa y valorar las formas alternativas de desarrollo endógeno, actualmente este particular territorio se deriva de una administración diaria de los recursos existentes en ellas, cada poblador ha incidido con sus actividades económicas, Martínez, (1994) argumenta que de forma individual la visión económica supone que los individuos agrupados en hogares, obtienen beneficios del ejercicio de variadas actividades, lo que repercute en la realidad colectiva en donde según Fonte M. y Ranaboldo, C. (2005), en este aspecto de gestión donde se obtiene productos colectivos.

Una vez tomado el tema económico se valora la dimensión humana y física del territorio, ya que ambos conducen a entender el grado de participación del hombre y la naturaleza en el afán de generar condiciones de vida, lo que es un motivante para que los hombres organizan su espacio, dicho asunto se contiene en la definición de las Ciencias Humanas, proceso del cual se destaca Humboldt (1845) argumentando la síntesis y localización como centro de la Geografía, la que está calificada como ciencia, posteriormente Ratzel (1914) aporta fundamentos naturalistas en la Geografía Humana, a lo que Reclus, E. (1965) afirma el espacio más allá de lo físico y lo presenta como acción combinada con las personas, dando perspectiva de lo natural y lo humano, el asunto en cuestión es tratado por Sorré, M. (1943) en su enfoque de la geografía como ecología humana, lo que es aplicable a la práctica de planificación territorial de toda localidad.

En la actualidad las cuencas han sido sujeto de estudio para el entendimiento de ese enfoque natural y social combinado, y en coherencia al riesgo ambiental mundial, se ha aplicado programas como Proyecto cuencas, con eje de formación ambiental no formal de carácter participativo, dicha experiencia comunitaria e institucional para Matagalpa es la que sirve de base para estás próximas líneas del documento presente.

Trascendental es aceptar que según la teoría aportada por tantos estudiosos sobre desarrollo territorial, brinda elementos que son comunes en la forma, estilo y ritmo de construcción del territorio y se engloba en la cultura, que retoma la identidad válida para esa sociedad en comunidad delimitada, el contexto en particular es una cuenca con sobresaliente actividad hidrográfica y 
agropecuaria, la que en la exigencia misma del sistema y medio de interacción diseña e instrumenta políticas orientadas al manejo integral y sostenible de los ecosistemas Sepúlveda (2002), de ello se califican en vitales tres recurso existentes en la cuenca como son suelo, agua, bosque, juntos son determinantes en la calidad del ámbito productivo, social, ambiental y económico de los habitantes.

Por ello ver a las cuencas de Matagalpa con sus potencialidades y vulnerabilidades, concentra la atención en los recursos naturales, así como los procesos hidrológicos y ecológicos que tienen calidad relativa a la calidad del manejo de la cuenca, si estos procesos de construcción del sistema van sin control sin lugar a dudas afectan la calidad del suelo y recursos hídricos dentro de la cuenca (Lal, 2000). En referencia a estas características mencionadas los riesgos experimentados ante fenómenos de origen antrópicos y naturales son los huracanes, los deslaves, incendios a causa de actividades agrícolas, afectando también el recurso forestal, así como el debilitamiento de la cobertura vegetal y las inundaciones. Todos estos efectos negativos sirven de base para promover en las comunidades de Molino Norte y Jucuapa el reconocimiento de la experiencia existente en la gestión adecuada y sostenible de los recursos naturales de estas cuencas, y valorando otras alternativas según los riesgos presentes.

Ahora bien en el contexto confluyen todos estos aspectos vinculados a la multidimensionalidad territorial, de tal forma que Molino Norte como Jucuapa según su realidad se reconoce el potencial sostenible, identificado en lo social, lo ambiental y lo económico, tanto así que es reconocible que en las últimas décadas se ha intensificado el mercado interno de consumo, con variada producción, todo viene de la explotación de los recursos naturales con que se cuenta Reyes, A. (2006), a esto se dice que Molino Norte según el Programa Socioambiental y de Desarrollo Forestal (POSAF), MARENA y Helsinki Consulting Group Ltd. HCG (2001) "es de mucha importancia puesto que el río Molino Norte aporta el 80 \% del consumo de agua potable de la ciudad de Matagalpa, la tercera ciudad más poblada de Nicaragua". (p. 2), y en cuanto a Jucuapa se destaca la actividad agropecuaria, por lo referido anteriormente ambas cuencas resultan activas en la economía del municipio.

De esto la función estratégica de las municipalidades se destaca por fomentar el ordenamiento territorial, el Gobierno Municipal ha diseñado un Plan Regulador de la Ciudad de Matagalpa, aprobado a través de ordenanza municipal $N^{\circ} 171204-28$ y publicado en la gaceta diario oficial. Actualmente el Plan Regulador está siendo implementado por el Departamento de Planificación Territorial de la municipalidad, este hecho denota que se está atendiendo desde la planificación el ordenamiento territorial tan necesario por eso tan vigente de análisis territorial en la segunda década del siglo XXI, lo que dio base para que surgiera el concepto de desarrollo sustentable, y según este es que se contiene el estudio a Matagalpa, ya se hacía referencia que la economía, así como la sociedad tienen estabilidad, por el contenido de trabajo u otros intereses particulares, en cambio la discusión se da desde el término de sustentable y equitativo con el tema ambiental, porque el estado de los recursos, la calidad de vida y el ambiente se conjuga con la productividad, 
eficiencia y eficacia, los que se ponen en riesgo si hace falta una conciencia ambiental, al hacer uso racional de los recursos naturales, válido de analizar en la generación presente y futura, donde el valor central es la equidad intergeneracional.

Según la estadísticas de pobreza, de enfermedades, de inequidad a los recursos, da origen a preocuparse, de aquí nacen planteamientos vinculados como este ¿Cómo atender la sustentabilidad de nuestras cuencas?, la respuesta es parte de la reflexión de Arríen (2010, p. 353), en nuestra cultura se trata y ve a la Madre Tierra como la proveedora permanente de bienes y recursos sin preocuparnos incluso de su desgaste casi irrecuperable, debido a que nuestra cultura occidental está fundamentada en el logos griego (la razón) y en el cogito cartesiano (pienso luego soy) es decir, nuestra cultura paga un alto tributo al logocentrismo y al racionalismo moderno fuente de la ciencia y sustento del desarrollo económico que necesita más y más de los recursos de la Tierra. Porque cuanto más le saquemos mayor desarrollo, mercado, comercio y consumo. Esta concepción y práctica se ha expresado en una acción permanente de abusar, deteriorar y destruir la Tierra hasta niveles universales preocupantes y con ellos amenazar la vida misma de la Humanidad, es decir, de nosotros mismos.

Actualmente se experimenta un cambio intenso en toda la naturaleza, Matagalpa con cambios climáticos y de temperatura que repercuten en la condición para la producción agrícola, otro efecto es la escasez de agua potable, y otros problemas, esta realidad no es ajena al país, en estudios ambientales se analiza y manifiesta claramente que la mayor causa es la poca responsabilidad del ser humano con los recursos naturales, tal como lo afirma Berenguer y Corraliza (2000, p. 1) el origen de muchos de los problemas ambientales, puede relacionarse con pautas del comportamiento humano y de la organización social, lo que vislumbra, de esto también se ha planteado una posible alternativa que Cone y Hayes (1982) emiten afirmando que muchos problemas ambientales tienen soluciones comportamentales y esta solución trae en si un alto nivel de concienciación y acción ambiental.

Es muy bueno que en caso Matagalpa, ya se tiene en las cuencas experiencias de programas de educación ambiental, las que han intentado atender el problema comportamental de sus habitantes, con acciones de capacitación, de divulgación a manera de educación informal, dichas intervenciones de los programas implican sesiones de trabajo que formen o desarrollen conciencia ambiental, de acuerdo a lo actuado en las cuencas se ha aplicado según (Godert 2005). La "prospectiva estratégica" con un proceso de reflexión que permite iluminar la toma de decisiones presentes a partir de posibles situaciones futuras.

En relación a la implementación de la prospectiva estratégica con la educación ambiental dada por proyecto cuencas en Matagalpa, se puede afirmar por un lado la relación de los funcionarios del proyecto con la comunidad, estimuló y logró vincular a muchas personas en las acciones consideradas válidas, partiendo de la toma de datos correspondientes a la acción de registrar las 
necesidades comunales mediante el levantamiento de información de las comunidades rurales (Colbert, 1999), sin embargo con los resultados de esta etapa, si se analiza la realidad de hoy, es necesario y urgente continuar con las acciones ante la comunidad, debido a que el riesgo ambiental que enfrentan por ejemplo con la escasez de agua, el deterioro del manto fértil, el despale, entre otros, es necesario determinar con la realidad de hoy y tomando como base lo antes actuado, un programa de educación ambiental, que deberá ser consensuado con los comunitarios.

Para enfocar la educación con el desarrollo en este siglo XXI, se plantea las siguientes pregunta ¿Cómo formular una estrategia de educación ambiental?, ¿Qué debe contener una estrategia de educación ambiental?, en las posibles respuestas se plantean ciertos requerimientos, por ejemplo el enfoque que debe basarse en promover la organización de los involucrados con capacidad de autogestión, así como la búsqueda de la equidad en sus relaciones internas y externas, ante estas potencialidades en el recurso humano, es posible utilizarlo para la construcción de una propuesta que conjugue experiencias, realidades y desafíos de hoy, ¿aplicará entonces el constructivismo social?, partiendo de que los constructos personales como lo expone en sus trabajos Kelly (1955), quien define el carácter del conocimiento como construcción y como producto de la acción humana Deval (1997), De ahí que se partirá en la construcción del programa con los presaberes de los comunitarios, basándose en la experiencia anterior de trabajo ambiental con ellos.

La historia de las cuencas Monino Norte y Jucuapa en cuanto a la educación ambiental de alguna manera tienen contenido, si este se valora a la luz de la pedagogía contemporánea, claramente se identifica teoría, metodología y práctica, que es insumo para el ambiente y el desarrollo, lo que a su vez se torna que lo que se aprende como algo nuevo, es incorporado a experiencias previas y a las estructuras mentales de cada persona, la que deposita esa información en una red de conocimientos y experiencias que existen previamente en el sujeto, en síntesis el aprendizaje tiene mucho de actividad y subjetividad, además se va modificando constantemente de acuerdo a las experiencias.

Efectivamente en el tema de aprendizajes, personas, naturaleza, economía, entre otros presentes en las cuencas, opinan Marúm-Espinoza y Reynoso-Cantú (2014), que la educación es un eje articulador en el desarrollo humano sustentable, siendo coherente a las intervenciones ambientales en las cuencas, es posible también incluir según Salazar Ordoñez y Sayadi, (2007) la innovación valorada como necesaria para el ritmo de evolución de los sistemas, lo que da cabida a proponer la Educación no formal con dinámica popular, según las experiencias esta práctica facilitaría rescatar el aspecto formativo que directamente incide en el crecimiento personal, en erradicar la pobreza que es parte de los compromisos adquiridos en el seno de la Organización de la Naciones Unidas (ONU, 2000), a través de los Objetivos del Milenio, la aplicación del programa de educación ambiental apunta a la ampliación de capacidades y libertades, como también fomentar la responsabilidad compartida quienes con sus interrelaciones sinérgicas (Reis, 2002: 116), con las cuales cada poblador explicita su voluntad, cuidan su trabajo con compromiso y de esta manera se educa con el ejemplo a las presentes y futuras generaciones de las cuencas de Matagalpa. 
Ahora la educación popular como práctica social, con dimensión educativa resurge como un espacio importante que promueve organización y participación, en las experiencias donde se ha aplicado profundamente ha transformado las conciencias individuales, consolidar esto es parte de lo clasificado como aprendizaje experiencial para el desarrollo de nuevas habilidades del pensamiento, el juicio crítico, donde es necesario pasar de la pasividad a la actividad, para ello es vital trabajar en los niveles de autoestima, de autoreconocimiento, presentes en la conciencia, tan importante para el tratamiento de los problemas, donde la disciplina, los valores y perspectivas están en la cultura, a través de ella el cambio será parte del patrimonio humano de los pobladores de la cuenca.

En orientación a ese resultado se toma a Ruvalcaba, (2010, p. 66). Donde la educación para el desarrollo sostenible (EDS) lleva implícita la noción de aplicación de programas adaptados al contexto local y culturalmente apropiados, de ahí que como orientación de la propuesta cabe valorar lo del diseño curricular enfocado a la educación no formal, popular, la que por su naturaleza apuntará al desarrollo de aprendizajes tan vitales como los que contempla en los pilares de la educación de Delors (1996) en su informe titulado "La educación encierra un tesoro", donde se plantea el paradigma de aprendizaje durante toda la vida como telón de fondo, basándolo en cuatro pilares: aprender a conocer, aprender a hacer, aprender a ser y aprender a vivir juntos (p. 34). Asimismo se reconoce que el concepto de educación a lo largo de la vida es la llave para entrar en el siglo XXI., de ahí que la gestión curricular concretizada en la organización y ejecución del proyecto pedagógico se retome los elementos del currículo los que según Coll, C. (1994) todo proceso educativo se puede orientar de acuerdo a estas preguntas ¿Para qué enseñar?, ¿Qué enseñar?, ¿Cuándo enseñar?, ¿Cómo y con qué enseñar?, ¿Qué, cuándo y cómo evaluar?, de acuerdo a todo lo anteriormente referido no todo en la formalidad aplica a la educación popular, y además refiere la pedagogía a utilizar de acuerdo a la población con la que se trabajará, en lo que casi es seguro, será con la andragogía debido a que es el conjunto de las técnicas de enseñanza orientadas a educar personas adultas.

La propuesta en sí requiere de una planificación que prevea en la consulta y consenso de intereses con la comunidad prever inquietudes, y adaptaciones necesarias para mejorar lo actual y diseñar elementos necesarios para el desarrollo de futuro.

En estas grandes líneas de la estructura de la propuesta no puede faltar el contenido expuesto por Gardner (2001)... mi colega de Harvard Davis Perkin quien destaca que la inteligencia se puede aprender en gran medida, permitiéndonos dominar varias estrategias, adquirir diversas capacidades $\mathrm{y}$ aprender a desenvolvernos en distintos contextos, precisamente el tema de las inteligencias múltiples es un referente del proceso que se puede vivir tomando lo que existe del contexto y asume el cultivo de la inteligencia como una posibilidad desde los mecanismos psicopedagógicos para motivar al aprendizaje natural, esto lleva a definir a detalle ¿De qué ha de ocuparse este programa de educación ambiental, no formal y de carácter popular?, si esto se aplica es un seguro 
cambio en la cultura de aprendizaje, ya que amarra sentidos, significados y expectativas válidos para el contexto y las resoluciones de los conflictos diarios, conscientemente define intenciones integrando de alguna manera el acto educativo/formativo orientado a su accionar cotidiano, Ávila (2001)

Todo este fundamento de referencias técnico, pedagógicas, si se aplican en las cuencas, desde, con y para las personas habitantes, se convertirían en parte del capital de dimensiones humanas, tan claves para el desarrollo sostenible rural, Según (Ruvalcaba, 2010) la educación ambiental es puente entre las ciencias naturales y las ciencias sociales, por lo que se puede decir que su enfoque es interdisciplinario, en ella caben todas las dimensiones territoriales, que serían las pautas para consultar y validarlas con los comunitarios, una vez vencido este paso se procede a desarrollar ideas del diseño del programa de educación ambiental, basado en las dimensiones territoriales y todos los aspectos referentes que están vinculados a la territorialidad, éstos en grandes aspectos se contienen en los siguientes tópicos que pueden ser constitutivos de unidades temáticas y aquí se presentan en este orden: I- Identidad (personal y territorial), II- Diagnóstico del territorio, caso particular la cuenca (descripción de la realidad en que viven-línea de base), III- Valoración de obstaculizadores o facilitadores que repercuten en la realidad territorial (desarrollo de conciencia y responsabilidad- axiología), IV- Elaboración en conjunto de planes de mejora con cronogramas de acción, V-Sistema o mecanismo de autoevaluación del proceso de desarrollo territorial, tomando la calidad ambiental y el papel de la comunidad en las acciones realizadas, VI- Sistema de trabajo colaborativo, políticas de estímulo, Políticas de fomento a la innovación y creatividad, formación de liderazgo comunitario, sentido de territorialidad, otros.

\section{CONCLUSIONES}

La educación es un arma liberadora como lo expresa Freire, no obstante debe ser usada con sumo cuidado para que cubra más que una transversalidad, eso implica que en la realidad territorial están

presentes las multidimensionalidades y las multiescalaridades, en las cuales el ser humano es vital condición.

La educación debe de ser planteada con gran fuerza en la no formalidad y la informalidad, ya que según su dinámica de ejecución, resulta ser complementaria y garantiza facilidades a las políticas de desarrollo, en particular las contempladas en Nicaragua.

La educación para el desarrollo territorial rural, constituye una idea y práctica a profundizar desde el Doctorado de Desarrollo Territorial Rural que sirve la UNAN FAREM Matagalpa, UNAN MANAGUA, las condiciones de formación permiten que sea una línea de investigación, la que en esta ocasión es asumido a través del diseño de una propuesta de programa de educación ambiental no formal y popular que implica el consenso con los actores comunitarios de las cuencas existentes en el municipio de Matagalpa. 
En el proceso de la construcción o diseño de dicho programa se tomará la teoría existente del desarrollo rural, para dimensionar la importancia que tiene de cara a la situación ambiental que se enfrenta, insistiendo en la necesidad de entender simultáneamente lo natural y lo humano que están íntimamente relacionado Miralles y Cebollada (2003), así como referirse a la formación humana como elemento clave para la aplicación de propuestas pertinentes al desarrollo territorial que se sustente en la práctica axiológica de cada habitante.

Se considera clave que este tipo de práctica de estudio con evidencias de aprendizaje que permitan formular alternativas para lograr hacer mejoras en los contextos sociorurales, es importante, ya que según la teoría del aprendizaje la significatividad está en que se conjuguen la teoría práctica, lo que es reflejado en la significatividad para la vida, además que la formación en todos los participantes en los niveles implicados en este proceso deben desarrollar conciencia y mostrar responsabilidad sobre el ambiente que le rodea.

\section{BIBLIOGRAFÍA}

Ausubel, D. P.: Novak, J. D,: Hanesian, H, (1983). Psicología Educativa, México: Trillas Education Psychology, Nueva York: Holt, Rinehart y Wiston.

Ávila, R. (2001) La cultura, modos de comprensión e investigación, Ediciones Antropos, Bogotá.

Batty, M. Agents, cells and cities: new representational models for simulating multi-scale urban dynamics. London: Centre for Advanced Spatial Analysis, Working Paper 65, 2003.

Colbert, V. (1999). Mejorando el acceso y la calidad de la educación para el sector rural pobre. El caso de la Escuela Nueva en Colombia. OEIEdiciones, Revista Iberoamericana de Educación, $\mathrm{N}^{\circ} 20$.

Coll, C. (1994) Psicología y Currículum. Una aproximación psicopedagógica a la elaboración del currículum escolar, Paidós, Barcelona.

Deval, J. (1997), Hoy todos son constructivistas. Cuadernos de Pedagogía, 257, 78-84.

Fonte, M., Ranaboldo, C. (2007) Desarrollo rural, territorios e identidades culturales. Perspectivas desde América Latina y la Unión Europea, Revista Opera, núm. 7 mayo, pp. 9-31 Universidad Externado de Colombia Bogotá, Colombia

García, A. (2000). La cuestión ambiental en la geografía del siglo XX, Anales de geografía de la Universidad Complutense, 20.

García, J. y Martinic, S. (1980), Intento de definición de Educación Popular Cide.

Gardner, H. (2001), La inteligencia reformulada, Editorial Paidós, Barcelona.

Humboldt, A. Von. (1845) Cosmos. Ensayo de una descripción física del mundo. Imprenta

Gaspar y Reig. Madrid.

Kelly, G. A. (1955), The Psychology of personal constructs, Nueva York; Norton.

Lal, R. (2000). Rationale for watersheds as a basis for sustaible management of soil and water resources. In Lal, R. (Ed) "Integrated watershed"

Lefebvre, Henri. The Production of Space. Cambridge: Blackwell Publishers, 1991 
Martínez, F. Access, the economic link in land use transport interaction. In: Valle Nevado, International Conference on Travel Behaviour Research, octubre 1994.

Marúm-Espinoza, E. y Reynoso-Cantú, E.L. (2014). La importancia de la educación no formal para el desarrollo sustentable en México. Revista Iberoamericana de Educación Superior (RIES), México, UNAM-IISUE/Universia, V (12), 137-155.

Miralles, C. (2002) Ciudad y transporte. El binomio imperfecto, Barcelona, Ariel

Ratzel, F. (1914) Geografía dell’Uomo (Antropo - geografia) Fratelli Bocca Editori. Torino. trad. de Ugo Cavallero

Reis, José (2002), “A globalização como metáfora da perplexidade? Os processos geo-económicos e o 'simples' funcionamento dos sistemas complexos", en Boaventura Sousa-Santos (coord.), A globalização e as ciências sociais, Cortez, São Paulo, pp. 109-134.

Reyes, A. (2006) Caracterización de las potencialidades y limitantes biofísicas de la subcuenca del río Jucuapa, Matagalpa - Nicaragua, Universidad Nacional Autónoma de Nicaragua - UNA.

Reclus, E. (1965) La geografía como metáfora de libertad. Plaza y Valdez S.A. México.

Salazar Ordoñez, M.; Sayadi, S. (2007). "La Revisión Intermedia de la PAC.

¿Acuerdo, desacuerdo o incertidumbre?’. Agricultura Familiar en España. Fundación de Estudios Rurales. pp. 48-58.

Santos, M. (1984). "The Redescovery and the remodeling of the planet in the technico-scientific period and New Roles of Sciences" International Social Science Journal 36-4. Paris: Unesco. 15 de mayo de 2009 < http://strates.revues.org/536>.

Santos, M. (1994). «O Retorno do Território», en Milton Santos, María Adélia A. de Souza y María Laura Silveira, coords., Território: Globalização e Fragmentação, pp.15-20, São Paulo, Hucitec-ANPUR.

Santos, M. (2000). La naturaleza del espacio. Técnica y tiempo. Razón y emoción, Barcelona, España, Editorial, Ariel Geografía. 348 p.

Sepúlveda, Sergio. 2002. Desarrollo Sostenible Microregional. Métodos para Planificación

Local. San José, IICA-UNA-CDT.

Sorre, M. (1943) Les fondements de la geographie humaine: Essai d'une ecologie de 1' homme. Armand Colin, Paris.

UNESCO. (2013, Enero). Una Mirada actual a la Educación encierra un Tesoro: evaluar la influencia del Informe Delors de 1996. Investigación y perspectivas en educación/UNESCO: Contribuciones temáticas, 4. 